\title{
Graphene/PSMA composite for gas sensing application
}

\author{
Hanis Camelia Muhar, Noor Syazwana Sahar², Fauzan Khairi Che Harun ${ }^{3}$, \\ Nurul Ashikin Abdul-Kadir ${ }^{4}$ \\ ${ }^{1,3,4}$ School of Electrical Engineering, Faculty of Engineering, Universiti Teknologi Malaysia, Johor Bahru, Malaysia \\ ${ }^{2}$ School of Biomedical Engineering and Health Sciences, Faculty of Engineering, Universiti Teknologi Malaysia, \\ Johor Bahru, Malaysia
}

\section{Article Info}

Article history:

Received Feb 7, 2020

Revised Apr 25, 2020

Accepted May 11, 2020

\section{Keywords:}

Conducting polymer

E-nose

Gas sensor

Graphene

Volatile organic compound

\begin{abstract}
This paper presents the fabrication of conducting polymer sensor which comprise of graphene-poly styrene-co-maleic acid (PSMA) composite that responds to volatile organic compound (VOC) via a change in the electrical resistance of the sensors. Five sensors composed of different material ratio were fabricated to find out the most prominent weight percentage ratio (wt \%) for optimum sensor response. Those materials were deposited onto silver electrode using drop-casting method. NI Card PCIE-6323 measures the voltage obtained from the sensor circuit and the resistance of the gas sensor monitored using LabVIEW instrument. It was observed from the experiments that combination of $60 \%$ graphene and $40 \%$ PSMA gives the highest sensor response.
\end{abstract}

This is an open access article under the CC BY-SA license.

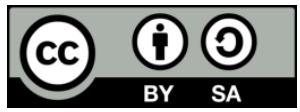

\section{Corresponding Author:}

Fauzan Khairi Che Harun,

School of Electrical Engineering,

Faculty of Electrical Engineering,

Universiti Teknologi Malaysia,

81310 UTM Johor Bahru, Johor, Malaysia.

Email: fauzan@utm.my

\section{INTRODUCTION}

Gas sensor refers to a device which can selectively respond to certain properties of the environment and can transfer this response into the electrical signals. This device has caught researchers' attention and has undergone a lot of development in the last few decades and now used to fulfill the industrial needs. Human nose is not suitable to be used as a gas sensor in industrial field since it is less accurate, and prone to fatigue [1]. Gas sensor in another hand has several advantages such as; it does not require properly trained personnel and not subject to individual breakdown or variation of sensitivity. In addition to that, once calibrated they can perform odor assessment on a continuous basis with a minimal cost and takes comparatively very little time. Studies on gas sensor had been conducted to widen the application for diverse purposes like medical diagnose [2-6], environmental control [7-11], and food industry [12-16]. The ability of gas sensor to measure, characterize, and discriminate between complex gas mixtures presents promising applications in those reported industries. Besides, research related to the gas sensor helps in development of multisensory communication. In current research, digital smell technology could let us transmit odors through the internet [17]. The process is by sending odor to the cloud using gas sensor, and regenerate the smell.

The common types of gas sensors that have attracted attention of many users including researchers and scientists in gas sensing field includes conducting polymer sensor, piezoelectric sensor, optical sensor, metal-oxide-semiconductor field-effect transistor (MOSFET) sensor, and conductivity sensor. Conducting polymer type gas sensor offers many advantages as compared to other types of gas sensor. Wide range of polymeric 
material available in the market allows a high discrimination in array sensor since different polymer gives different response to a given odorant. Besides, this type of gas sensor requires a simpler fabrication technique, signal reproducibility, and enhanced sensitivity [18]. Conducting polymer sensor works using percolation theory principle. As the polymer composite sensor is exposed to vapor sample, some of the vapor permeates into the polymer, causing the polymer to expand. This expansion reduces the number of conducting pathway for charge carriers. Subsequently, this polymer increases the electrical resistance of the polymer composite [19].

This paper studies the effort that has been carried out to fabricate five conducting polymer sensors compose of graphene-polystyrene-co-maleic acid (PSMA) composite that compound volatile organic compounds (VOC). Graphene nanomaterial shows great promise for applications in nano-electrics, flexible electronics, sensors, and solar cells [20]. However, the percentage of combination between graphene and polymers that gives the best performance has not yet been identified. It is necessary to find the ideal weight percentage responds to volatile organic ratio of the composite for future development of gas sensor.

\section{SENSOR FABRICATION AND MEASUREMENT}

To analyze performance of the sensor, several experiments were performed. The block diagram of the whole setup is demonstrated in Figure 1, which consists of sensor chamber, bubbler, National Instrument (NI) my-DAQ, pump, power supply, and computer. Sample gas was tested on the sensors which located inside the metal chamber. The pump flushed the odor vapor through inlet tube. By using interface circuitry, sensor was connected to the computer via NI my-DAQ

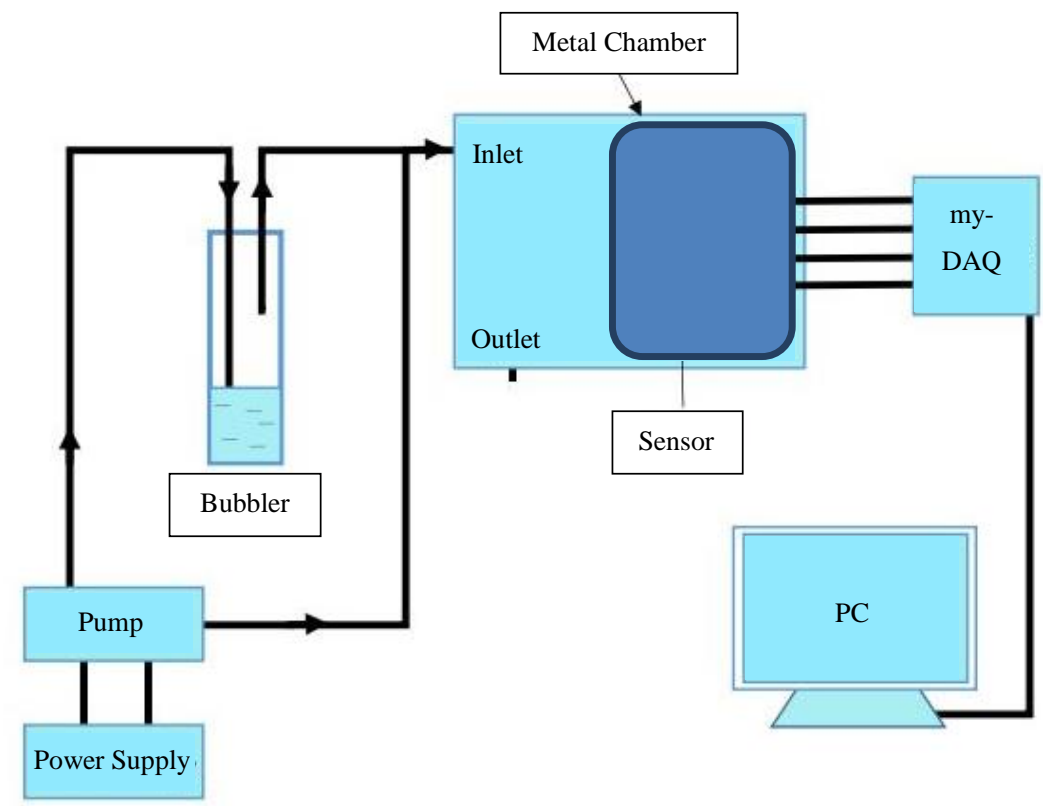

Figure 1. Schematic diagram of experiment

In order to measure resistance changes upon odor exposure, sensor circuit which consists of Wheatstone Bridge was designed. When the bridge is fully balanced, the right-side resistors which are identical to the left side resistors $(\mathrm{R} 1=\mathrm{R} 2$, and $\mathrm{R} 3=\mathrm{Rx})$, and the voltage across the bridge is zero. However, with a small change in resistance on one of the resistors, the bridge becomes unbalanced, and a voltage difference appears. This feature of Wheatstone Bridge makes it suitable to be used as measuring circuit for sensors. The sensing material was deposited onto the interdigitated electrode using drop casting technique up to five layers, but it cannot be seen considering the color of grapheme-PSMA mixture is crystal clear.

\subsection{Chemical preparation}

Ultrapure pristine graphene monolayer flakes (dispersion in ethanol) with concentration of $1 \mathrm{mg} / \mathrm{L}$ were procured from graphene supermarket containing average flake thickness $0.35 \mathrm{~nm}$, and average particle (lateral) size $\sim 550 \mathrm{~nm}(150-3000) \mathrm{nm}$. PSMA (Mw=65,000) was purchased from Sigma-Aldrich. Since PSMA is in solid form, it needs to be dissolved with ethanol. The ratio of graphene weight is too small 
as compared to PSMA weight. Hence PSMA solution was diluted for two times, to ensure that PSMA weight is small enough to be mixed with graphene. Polymer solutions were prepared from $10 \mathrm{mg}$ PSMA and $10 \mathrm{ml}$ ethanol. After being completely dissolved, $1 \mathrm{ml}$ of the mixture was added to $9 \mathrm{ml}$ ethanol to dilute the solution. They were homogeneously dispersed by physical agitation for 30 minutes with the aid of digital ultrasonic cleaner at room temperature. Graphene was used as received without any further treatment. Five types of sensor were fabricated with different graphene/PSMA ratio. The identification name of each sensor and their respective ratio are listed in Table 1. Amount of material to be deposited was calculated based on its weight percentage (wt \%), where:

$$
\text { weight percentage }(w t \%)=\frac{\text { weight of solute }}{\text { weight of solution }} \times 100 \%
$$

Table 1. Ratio and quantity of composite material

\begin{tabular}{lcccc}
\hline Label & Graphene ratio $(\%)$ & Graphene quantity $(\mathrm{ml})$ & PSMA Ratio $(\%)$ & PSMA volume (ul) \\
\hline Sensor A & 70 & 0.5 & 30 & 2.1 \\
Sensor B & 60 & 0.5 & 40 & 3.3 \\
Sensor C & 50 & 0.5 & 50 & 5.0 \\
Sensor D & 30 & 0.5 & 70 & 12 \\
Sensor E & 20 & 0.5 & 80 & 20 \\
\hline
\end{tabular}

Drop casting technique was deployed using micro pipette with different ranges. Two ul of the solution was deposited on a sensor substrate with an active area $(0.25$ " $\times 0.25$ ") consisting of Au interdigitated electrodes. This process was repeated until resistance was between $3 \mathrm{M}$ ohm and $9 \mathrm{M}$ ohm. As-deposited composite films were dried at room temp for 1 hour. Figure 2 shows the complete setup for this experiment. Sensor was placed into a metal chamber. Microdiaphragm pump (Model NMP 30 KNDC, KNF Neuberger Inc., Trenton, NJ) was connected by polyethylene tubing to a bubbler. A laboratory DC power supply was used to supply $5 \mathrm{~V}$ voltage, and data acquisition hardware (NI, my-DAQ) was used to measure the voltage drops across the sensors.

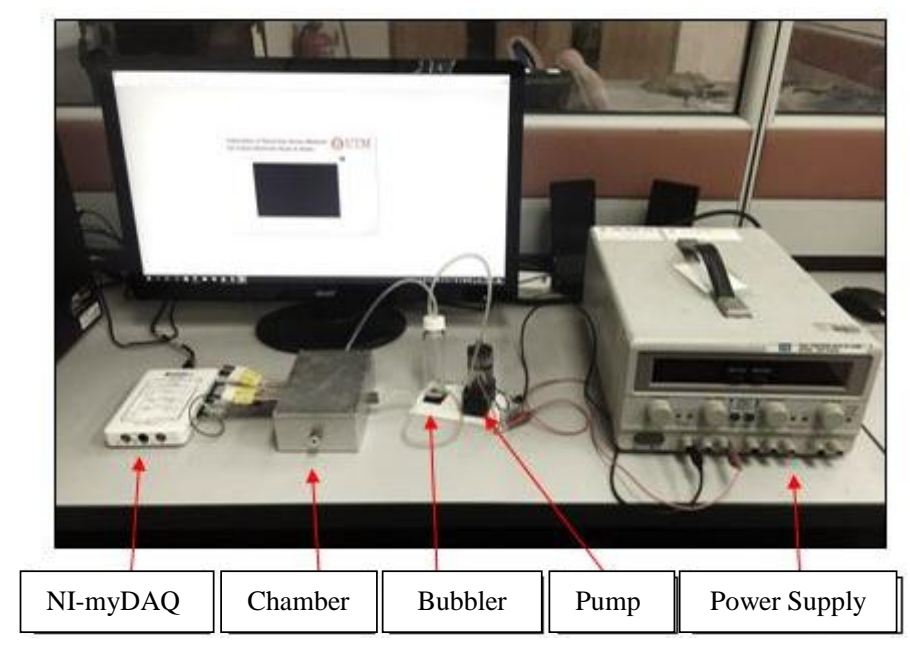

Figure 2. Complete setup of experiment

\subsection{Experimental procedure}

In this experiment, Pucelle perfume was tested as the vapor samples, and environment air was used as the baseline. This sensor was designed for gas sensor application, and hence VOC was tested instead of identifying single compound. $3 \mathrm{~mL}$ of the vapor samples were transferred to screw-capped $10 \mathrm{~mL}$ bottles bubbler. The pump flown the reference gas to the chamber for 20 seconds to create a baseline voltage, followed by gas under test for 30 seconds, and flow reference gas to clean the chamber for 20 seconds. The perfume concentration was controlled in each experiment by delivering the same pump voltage specifically $5 \mathrm{~V}$. The time gap between each repetition is set to 5 minutes, and the same sensor was deployed in each repetition. The baseline voltage for all five sensors is $2.5 \mathrm{~V}$. 


\section{RESULTS AND DISCUSSION}

Each sensor was deposited with graphene/PSMA composite and with different weight percentage (wt \%). To test the sensor performance, Pucelle perfume was utilized as the subject for this experiment. The response magnitude for each sensor was observed to investigate graphene/PSMA sensing capability towards odorant. In addition, the response time and recovery time for each sensor was determined and discussed. The fractional baseline manipulation was calculated to verify which sensor gives the best response towards Pucelle perfume.

\subsection{Characteristics of graphene/PSMA sensor}

In each experiment, three readings were taken and named as Repetition 1, Repetition 2, and Repetition 3. From Figure 3, Repetition 1 was labeled with blue colored line, Repetition 2 labeled with orange colored line, and Repetition 3 labeled with grey colored line. The voltage measurement of sensor deposited with $70 \%$ Graphene $(0.5 \mathrm{ml})$ and 30\% PSMA (2.1 ul) labelled as Sensor A has been carried out with three repetitions and the results are shown in Figure 3.

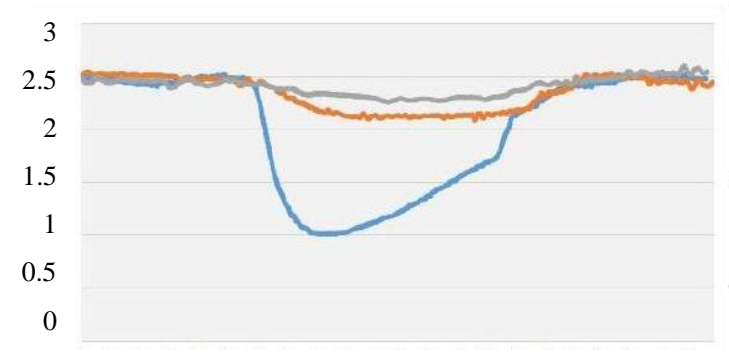

(a)

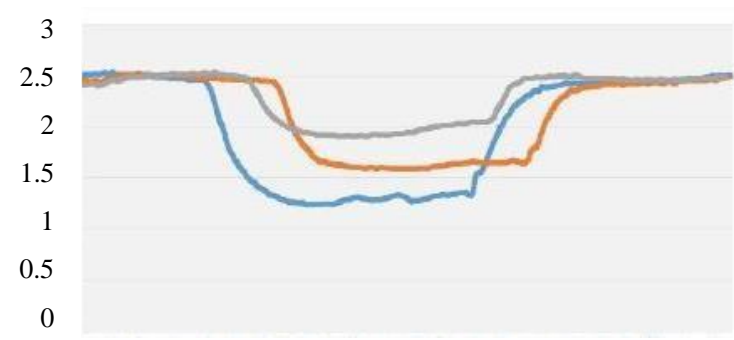

(c)

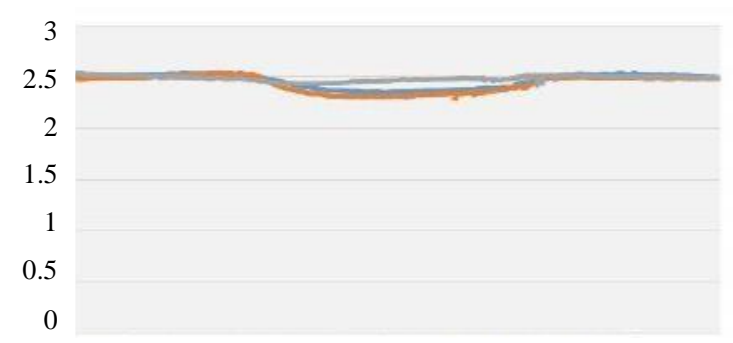

(e)

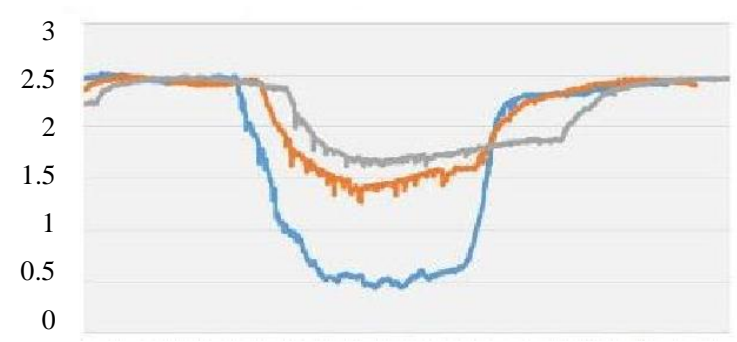

(b)

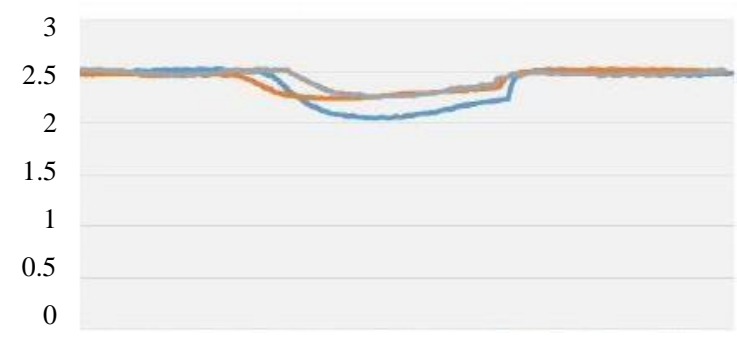

(d)
Repetition 1

Repetition 2

Repetition 3

Figure 3. The characteristics of five sensors, (a) Sensor A (70 \% graphene, 30\% PSMA), (b) Sensor B (60\% Graphene, 40\% PSMA), (c) Sensor C (50\% graphene, 50\% PSMA), (d) Sensor D (30\% graphene, 70\% PSMA), (e) Sensor E (20\% graphene, $80 \%$ PSMA)

From Figure 3(a), the voltage achieves the highest peak of $1 \mathrm{~V}$ when exposed to perfume vapor and drop to its baseline value $(2.5 \mathrm{~V})$ as reference air was flushed into it. According to [21], the general experimental voltage reading should rise when exposed for testing gas and slump as reference gas is flushed into the chamber. In this experiment, the first repetition illustrates a slight decline before the reference gas is flushed into the chamber. This fall off may due to graphene/PSMA structure itself. As the graphene content in the composite is dominant, a small expansion of polymer would disrupt the conducting pathway between the charge carriers and hence increase the electrical resistance between electrodes. Besides, this reduction 
may occur due to sensor poison. The response time, recovery time, and sensitivity of each repetition was calculated and recorded in Table 2. Repetition 1 has the shortest response time $(91 \mathrm{~s})$ as compared to Repetition 2 (129 s) and Repetition 3 (177 s). However, it takes a longer time to reach baseline value when flushed with reference air $(165 \mathrm{~s})$. The sensitivity is calculated to be 0.6 , which is much greater than Repetition 2 (0.17) and Repetition 3 (0.10).

Table 2. Characteristics of Sensor A

\begin{tabular}{llll}
\hline Repetition & Response time (s) & Recovery time (s) & Sensitivity (\%) \\
\hline 1 & 91 & 165 & 0.60 \\
2 & 129 & 67 & 0.17 \\
3 & 177 & 154 & 0.10 \\
\hline
\end{tabular}

Responds for Sensor B which deposited with $60 \%$ graphene $(0.5 \mathrm{ml})$ and $40 \%$ PSMA (3.3 ul) was illustrated in Figure 3(b). For Repetition 1, the voltage dropped to $0.43 \mathrm{~V}$ when exposed to perfume vapor. This sensor shows the largest voltage difference $(2.07 \mathrm{~V})$ as compared to the other sensors. Hence, it was considered as the most sensitive sensor for detection of Pucelle perfume. The summary of response time, recovery time, and sensitivity of each repetition was shown in Table 3. Although Repetition 1 has a longer response time (112 s) as compared to Repetition 3 (99 s), it still gives the highest sensitivity (0.83). As for recovery time, Repetition 3 shows the fastest (179s) and Repetition 1 is the slowest (217 s).

Table 3. Characteristics of Sensor B

\begin{tabular}{|c|c|c|c|}
\hline Repetition & Response time (s) & Recovery time (s) & Sensitivity (\%) \\
\hline 1 & 112 & 217 & 0.83 \\
\hline 2 & 124 & 188 & 0.44 \\
\hline 3 & 99 & 179 & 0.33 \\
\hline
\end{tabular}

The voltage measurement of sensor deposited with $50 \%$ Graphene $(0.5 \mathrm{ml})$ and $50 \%$ PSMA (5 ul) labelled as Sensor $\mathrm{C}$ had been carried out with three repetitions and the result is shown in Figure 3(c). The voltage achieved the highest peak of $1.2 \mathrm{~V}$ when exposed to perfume vapor and drop to its baseline value $(2.5 \mathrm{~V})$ as reference air was flushed into the chamber. The voltage difference for Repetition 1 is $1.27 \mathrm{~V}$. The summary of response time, recovery time, and sensitivity of each repetition was shown in Table 4 . For response time, Repetition 2 shows the shortest (52 s), followed by Repetition 3 (76 s), and Repetition 1 (107 s). As for recovery time, the shortest is Repetition 3 (46 s), followed by Repetition 2 (206 s), and Repetition 1 (248 s). However, Repetition 1 shows the highest sensitivity (0.51) as compared to Repetition 2 and 3.

Table 4. Characteristics of Sensor C

\begin{tabular}{llll}
\hline Repetition & Response time (s) & Recovery time (s) & Sensitivity (\%) \\
\hline 1 & 107 & 248 & 0.51 \\
2 & 52 & 206 & 0.37 \\
3 & 76 & 46 & 0.24 \\
\hline
\end{tabular}

Figure 3(d) shows the result for Sensor D which deposited with $30 \%$ graphene $(0.5 \mathrm{ml})$, and $70 \%$ PSMA (12 ul). For Repetition 1, the respond fell from $2.5 \mathrm{~V}$ to $2.04 \mathrm{~V}$, which shows a voltage difference of $0.46 \mathrm{~V}$. The summary of response time, recovery time, and sensitivity of each repetition was shown in Table 5. Repetition 3 has the shortest response time (87 s), followed by Repetition 2 (96 s), and Repetition 1 (133s). However, Repetition 1 has the shortest recovery time (27 s) and the highest sensitivity (0.18).

Table 5. Characteristics of Sensor D

\begin{tabular}{llll}
\hline Repetition & Response time (s) & Recovery time (s) & Sensitivity (\%) \\
\hline 1 & 133 & 27 & 0.18 \\
2 & 96 & 44 & 0.11 \\
3 & 87 & 55 & 0.10 \\
\hline
\end{tabular}


Figure 3(e) shows the result for Sensor E which deposited with $20 \%$ graphene $(0.5 \mathrm{ml})$, and $80 \%$ PSMA (20 ul). The voltage for Repetition 1 reduced from $2.5 \mathrm{~V}$ to $2.33 \mathrm{~V}$, which shows a voltage difference of $0.17 \mathrm{~V}$. The summary of response time, recovery time, and sensitivity of each repetition was shown in Table 6. Repetition 1 has the longest response time (169 s) as compared to Repetition 2 (110 s) and Repetition 3 (51 s). It has the longest recovery time (83 s) as well. The sensitivity is not much different from Repetition 2 and Repetition 3.

Table 6. Characteristics of Sensor E

\begin{tabular}{llll}
\hline Repetition & Response time (s) & Recovery time (s) & Sensitivity (\%) \\
\hline 1 & 169 & 83 & 0.07 \\
2 & 110 & 78 & 0.09 \\
3 & 51 & 54 & 0.04 \\
\hline
\end{tabular}

Figure 4 illustrates the response magnitude of five kinds of sensors when exposed to Pucelle perfume. Each colored line represents a separate sensor output from each sensor type. Sensor A represented by the green colored line, red colored line represents Sensor B, while yellow colored line represents Sensor C. Sensor D represented by purple colored line and Sensor E represented by blue colored line. Generally, each sensor shows similar trend which comparable to each other.
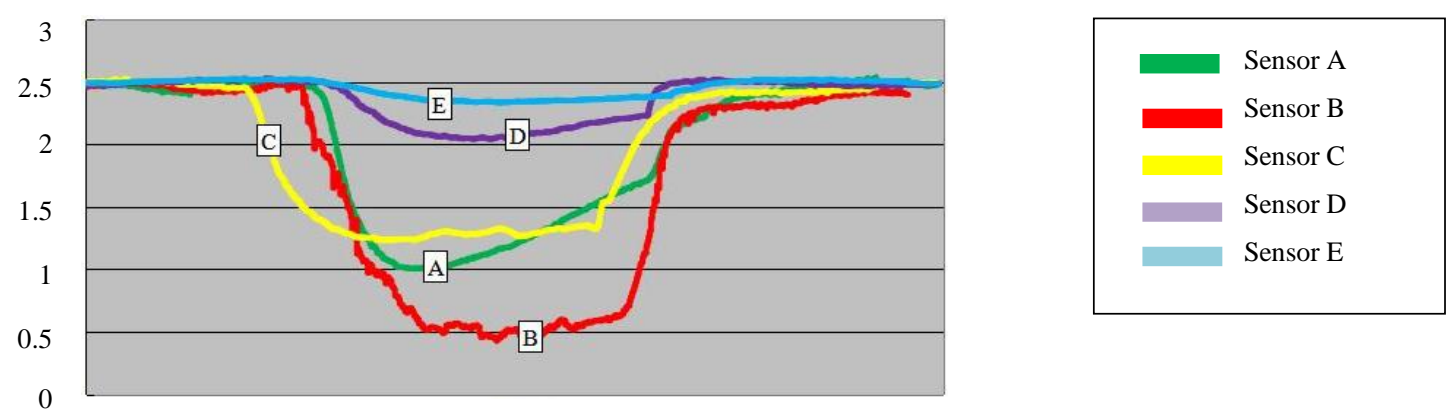

Figure 4. Comparison of five sensors

It can be clearly seen from the graph that different sensors provide different response towards the same odorant. Each sensor proved the capability of graphene/PSMA based sensor to detect presence of Pucelle perfume. However, their sensitivity, response time and recovery time varied due to different weight percentage (wt \%) of graphene and PSMA mixture. The resistivity of each sensor results from the expansion of polymer when exposed to odorant. This expansion reduces the number of conducting pathways for charge carriers that naturally present in this composite. For this reason, the weight percentage (wt \%) of polymer in the graphene-polymer composite consequently affect the resistivity of the sensor. As reported in [19], responds of each sensor are different for different odorant. Some of the sensors might be more sensitive for specific odorant but less sensitive for another odorant. By comparing all five sensors in this experiment, sensor B which deposited with $60 \%$ graphene and 40\% PSMA gives the highest sensor response towards Pucelle perfume. It could be concluded as the dominant sensor to detect the presence of Pucelle perfume's vapor. However, this sensor might not be the best sensor to detect another odorant.

Sensor A which deposited with 70\% graphene and 30\% PSMA has the steepest gradient of response compared to the other four sensors, which means Sensor A has the fastest response time towards perfume vapor (91 s). It was then followed by Sensor C (107 s), Sensor B (112 s), Sensor D (133 s) and Sensor E (169 s). As for recovery time, Sensor D has shortest time (27 s), followed by Sensor E (83 s), Sensor A (165 s), sensor B (217 s), and Sensor C (248 s). The summary of response time, recovery time, and sensitivity for each sensor was reported in Table 7.

Table 7. Summary for each sensor response

\begin{tabular}{llll}
\hline Sensor & Response time (s) & Recovery time (s) & Sensitivity (\%) \\
\hline A & 91 & 165 & 0.60 \\
B & 112 & 217 & 0.83 \\
C & 107 & 248 & 0.51 \\
D & 133 & 27 & 0.18 \\
E & 169 & 83 & 0.07 \\
\hline
\end{tabular}




\section{CONCLUSION}

Utilization of graphene/PSMA based gas sensor in this study has provided sufficiently strong sensor response to detect organic volatile compound. In addition, results from experiments demonstrated that combination of $60 \%$ graphene and $40 \%$ PSMA gives the highest sensor response for measurement of the subject. This result was concluded prior to three times experiment repetition.

As an extension to improve and optimize the sensor system, it would be interesting to diversify the type of polymer to produce a new sensor. Compilation of multiple weight percentage of graphene with different polymers may be investigated in future works, to find out whether $60 \%$ graphene and $40 \%$ PSMA combination still works for another graphene-polymer combination. In term of fabrication technique, problem arises when graphene acquired from the market has a very low concentration. It is difficult to quantify the polymer volume in order to obtain the desired wt \% ratio. Therefore, in future research graphene may be produced via other methods such as Hummers Offeman's method which has been used by another researcher [22, 23]. In another hand, condition of the experiment should be strictly maintained to get a more accurate result. In particular, the humidity and flow pressure should be continuously monitored. Sensitivity of the sensor significantly affected as this control keeps changing. Hence to overcome this problem, a humidity sensor, and pressure sensor should be installed inside the sensor chamber in future research. Besides, inkjet printing technique should be implemented to replace drop-casting technique. Although using this technique is quite complex and expensive, it would give a very high precision material deposition process and able to control the deposition process in a more detailed way. Li et al. [24] and Secor et al. [25] in their research use this inkjet printing method for deposition of graphene. This inkjet printing technology allows a wide range of substrate includes cloth and transparent paper. Hence, a flexible graphene-based gas sensor could be fabricated in the future study.

\section{ACKNOWLEDGEMENTS}

The authors would like to thank Ministry of Higher Education Malaysia and Universiti Teknologi Malaysia for funding this study under Matching Grant vot No. Q.J130000.3001.01M13 and GUP Tier 2 vot No. Q.J130000.2651.17J08.

\section{REFERENCES}

[1] A. D. Wilson and M. Baietto, “Applications and advances in electronic-nose technologies," Sensors, vol. 9, no. 7 pp. 5099-5148, 2009

[2] L. Kunos, A. Bikov, Z. Lazar, B. Z. Korosi, P. Banedek, G. Losonczy, and I. Horvath, "Evening and morning exhaled volatile compound patterns are different in obstructive sleep apnoea assessed with electronic nose," Sleep and Breathing, vol. 19, pp. 247-253, 2015.

[3] A. Roine, E. Veskimäe, A. Tuokko, P. Kumpulainen, J. Koskimäki, T. A. Keinänen, M. R. Häkkinen, J. Vepsäläinen, T. Paavonen, J. Lekkala, T. Lehtimäki, T. L. Tammela, and N. K. J. Oksala, "Detection of prostate cancer by an electronic nose: a proof of principle study," The Journal of Urology, vol. 192, no. 1, pp. 230-235, 2014.

[4] O. Sibila, L. Garcia-Bellmunt, J. Giner, J. L. Merino, G. Suarez-Cuartin, A. Torrego, I. Solanes, D. Castillo, J. L. Valera, B. G. Cosio, and V. Plaza, "Identification of airway bacterial colonization by an electronic nose in chronic obstructive pulmonary disease," Respiratory Medicine, vol. 108, no. 11, pp. 1608-1614, 2014.

[5] K. de Heer, M. P. van der Schee, K. Zwinderman, I. A. H. van den Berk, C. E. Visser, and R. van Oers, P. J. Sterk, "Electronic nose technology for detection of invasive pulmonary aspergillosis in prolonged chemotherapy-induced neutropenia: a proof-of-principle study," Journal of Clinical Microbiology, vol. 51, no. 5, pp. 1490-1495, 2013.

[6] T. Greulich, A. Hattesohl, A. Grabisch, J. Koepke, S. Schmid, S. Noeske, C. Nell, M. Wencker, R. A. Jörres, C. F. Vogelmeier, U. Köhler, and A. R. Koczulla, "Detection of obstructive sleep apnoea by an electronic nose," European Respiratory Journal, vol. 42, no. 1, pp. 145-155, 2013.

[7] L. Zhang, F. Tian, S. Liu, J. Guo, B. Hu, Q. Ye, L. Dang, X. Peng, C. Kadri, and J. Feng, "Chaos based neural network optimization for concentration estimation of indoor air contaminants by an electronic nose," Sensors and Actuators A: physical, vol. 189, pp. 161-167, 2013.

[8] L. Zhang, F. Tian, C. Kadri, B. Xiao, H. Li, L. Pan, and H. Zhou, "On-line sensor calibration transfer among electronic nose instruments for monitoring volatile organic chemicals in indoor air quality," Sensors and Actuators B: Chemical, vol. 160, no. 1, pp. 899-909, 2011.

[9] L. Zhang, F. Tian, H. Nie, L. Dang, G. Li, Q. Ye, and C. Kadri, "Classification of multiple indoor air contaminants by an electronic nose and a hybrid support vector machine," Sensors and Actuators B: Chemical, vol. 174, pp. 114-125, 2012.

[10] T. Sobański, A. Szczurek, K. Nitsch, B. W. Licznerski, and W. Radwand, "Electronic nose applied to automotive fuel qualification," Sensors and Actuators B: Chemical, vol. 116, no. 1-2, pp. 207-212, 2006.

[11] J. W. Gardner, H. W. Shin, E. L. Hines, and C. S. Dow, "An electronic nose system for monitoring the quality of potable water," Sensors and Actuators B: Chemical, vol. 69, no. 3, pp. 336-341, 2000.

[12] S. Qiu, J. Wang, and L. Gao, "Qualification and quantisation of processed strawberry juice based on electronic nose and tongue," LWT-Food Science and Technology, vol. 60, no. 1, pp. 115-123, 2015. 
[13] J. P. Santos and J. Lozano, "Real time detection of beer defects with a hand held electronic nose," 2015 10th Spanish Conference on Electron Devices (CDE), pp. 1-4, 2015.

[14] M. Kukade, A. J. Moshayedi, and D. C. Gharpure, "Electronic-nose (E-nose) for recognition of cardamom, nutmeg and clove oil odor," Electronics and its Interdisciplinary Applications (NCAEIA-2014), vol. 2, 2014.

[15] J. Gruber, H. M. Nascimento, E. Y.Yamauchi, R. W. C. Li, C. H. A. Esteves, G. P. Rehder, C. C. Gaylarde, and M. A. Shirakawa, "A conductive polymer based electronic nose for early detection of Penicillium digitatum in post-harvest oranges," Materials Science and Engineering: C, vol. 33, no. 5, pp. 2766-2769, 2013.

[16] X. Hong, J. Wang, and Z. Hai, "Discrimination and prediction of multiple beef freshness indexes based on electronic nose," Sensors and Actuators B: Chemical, vol. 161, no. 1, pp. 381-389, 2012.

[17] K. Karunanayaka, S. K. Ufere, H. Camelia, and A. D. Cheok, "Electric stimulation of human nasal conchae towards reproduction of olfactory sensations," Clinical Medical Image Library, vol. 4, vol. 5, pp. 108-1-2, 2018.

[18] K. Madgula, and L. N. Shubha, "Conducting polymer nanocomposite-based gas sensors," Functional Nanomaterials. Springer, Singapore, pp. 399-431, 2020

[19] K. Arshak, E. Moore, G.M. Lyons, J. Harris, and S. Clifford, "A review of gas sensors employed in electronic nose applications," Sensor review, vol. 24, no. 2, pp. 181-198, 2004.

[20] R. S. Bhoria, "Graphene: Potential material for nanoelectronics applications," Indian Journal of Pure \& Applied Physics (IJPAP), vol. 53, no. 8, pp. 501-513, 2015.

[21] N. H. Mahmood, A. M. Jumadi, and F. K. Che Harun, "Gas Sensor based on carbon black polymer composite for electronic nose," ELEKTRIKA-UTM Journal of Electrical Engineering, vol. 13, no. 2, pp. 1-7, 2012.

[22] T. Alizadeh and L. Hamedsoltani, "Graphene/graphite/molecularly imprinted polymer nanocomposite as the highly selective gas sensor for nitrobenzene vapor recognition," Journal of Environmental Chemical Engineering, vol. 2, no. 3, pp. 1514-1526, 2014

[23] T. Alizadeh and L. Hamed Soltani, "Graphene/poly (methyl methacrylate) chemiresistor sensor for formaldehyde odor sensing," Journal of Hazardous Materials, vol. 248, pp. 401-406, 2013.

[24] J. Li, F. Ye, S. Vaziri, M. Muhammed, M. C. Lemme, and M. Östling, "Efficient inkjet printing of grapheme," Advanced Materials, vol. 25, no. 29, pp. 3985-3992, 2013.

[25] E. B. Secor, P. L. Prabhumirashi, K. Puntambekar, and M. C. Hersam, "Inkjet printing of high conductivity, flexible graphene patterns," The Journal of Physical Chemistry Letters, vol. 4, no. 8, pp. 1347-1351, 2013.

\section{BIOGRAPHIES OF AUTHORS}
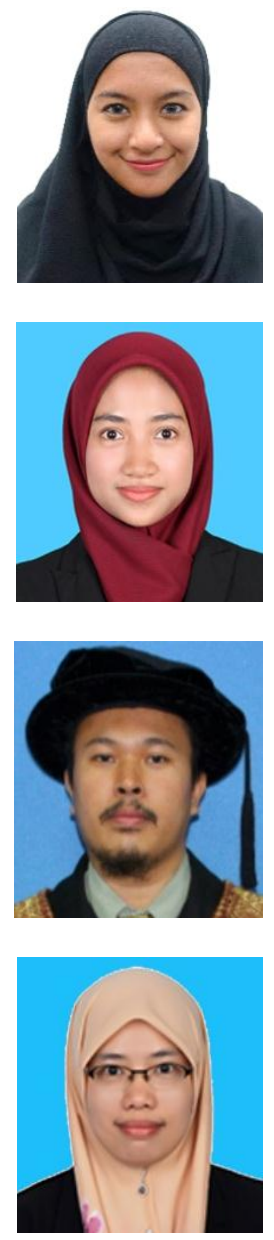

Hanis Camelia Muhar received her Bachelor's Degree in Electrical (Medical Electronics) Engineering from Universiti Teknologi Malaysia in 2014. Currently, she is a Ph.D candidate in Electrical Engineering at Universiti Teknologi Malaysia. Her research area related to electronic circuit and system.

Noor Syazwana Sahar received her Bachelor's Degree in Electrical (Medical Electronics) Engineering from Universiti Teknologi Malaysia in 2014. Previously, she worked as Product Engineer in Freescale Semiconductor, Petaling Jaya. Currently, Syazwana pursuing her Master of Philosophy study in Biomedical Engineering at Universiti Teknologi Malaysia. Her research area related to bio-signal processing of ECG and electronic circuit.

Fauzan Khairi Che Harun received his BEng degree in Electrical-Electronics Engineering from Universiti Teknologi Malaysia in 2003. He then further his study in the area of biomedical engineering in University of Warwick where he was awarded with MSc in Advanced Electronic (2005) and $\mathrm{PhD}$ in Engineering (2010). His research interests are mainly but not restricted to biomedical electronics, Biological Inspired Micro-system and Electronic Nose System. Currently he is a senior lecturer at the School of Electrical Engineering, UTM.

Nurul Ashikin Abdul-Kadir serves at Universiti Teknologi Malaysia in the field of Electrical and Electronic Engineering as Senior Lecturer since 2019. She received her master and phD degree in Electrical Engineering and her bachelor degree in Electrical Engineering (Medical Electronics). Her research interest is related to electronic circuit, bio-signal processing (ECG, EEG, EMG), embedded electronics and pattern recognition. 